\title{
849 RESTORE T CELL ENGAGER PLATFORM DEPLETES MDSC IN PARALLEL WITH ANTIGEN-SPECIFIC SOLID TUMOR CYTOTOXICITY
}

Sterling Eckard*, Bianca Rojo, Li Mei, Alberto Ponce, Patrick Chun, Victoria Smith. Amphivena Therapeutics, South San Francisco, CA, USA

Background Amphivena's ReSTORE T cell engager platform produces tunable, potent, and selective molecules that mitigate immune suppression while also directly targeting tumor cells. The core function of this platform utilizes AMV564, a clinically active molecule that has been shown in a phase 1 study to selectively deplete myeloid-derived suppressor cells (MDSC) and produce clinical responses in cancer patients (NCT04128423). ${ }^{1}$ Increased levels of MDSC in cancer patients correlate with reduced overall survival, as MDSC suppressive factors impair $\mathrm{T}$ cell activation and anti-tumor immunity. With the addition of unique target-specific $\mathrm{VHH}$ domains to the AMV564 core, new platform molecules can be engineered with additional functionality to selectively target tumor-specific antigens.

Methods Cell lines, primary human cells, and patient samples were analyzed using flow cytometry with appropriate marker panels including directly labeled AMV564 (phycoerythrin) and labeled anti-AMV564 antibodies. $\mathrm{T}$ cell cytotoxicity assays were conducted using primary human $\mathrm{T}$ cells and target cells (3:1 ratio) for 72 hours. Biophysical characterization was performed using standard techniques with SDS-PAGE gels, analytical SEC, and Octet analysis.

Results We have engineered a stable and potent $\mathrm{T}$ cell engager platform that targets both MDSC and tumor cells (figure 1). The tumor antigen potency relative to MDSC cytotoxicity is tunable, allowing design flexibility. We demonstrate that ReSTORE molecules expressing a variety of $\mathrm{VHH}$ domains can effectively mediate a cytolytic synapse with KG-1 target cells, which represent MDSC in in vitro assays due to their CD33 expression level and myeloid differentiation state (figure 2A). ${ }^{2}$ Our platform molecules act as robust $\mathrm{T}$ cell agonists while maintaining selectivity with minimal activation-induced cell death (AICD) compared to positive controls (CD3/CD28) and demonstrate dose-dependent enhancement of cytolytic activity (figure 2B). Molecules with targets ranging from tumor targets to antiviral epitopes have been developed, and they maintain potent and selective $\mathrm{T}$ cell-dependent cytotoxicity against cells expressing the target antigen (figure 3A). New targets can be engineered to accommodate desired potency, and bivalent design yields selectivity (figure 3B). In all cases, these molecules significantly inhibit cancer cell growth in a dose dependent manner.

\section{$R-T-M, M-T-R$}

Abstract 849 Figure 1 ReSTORE platform molecules are bivalent and trispecific. The core function of each molecule targets MDSC (M) while activating and expanding T cells (T). By adding unique VHH domains, these molecules gain the ability to target specific tumor antigens (R) and direct specific tumor cell killing.

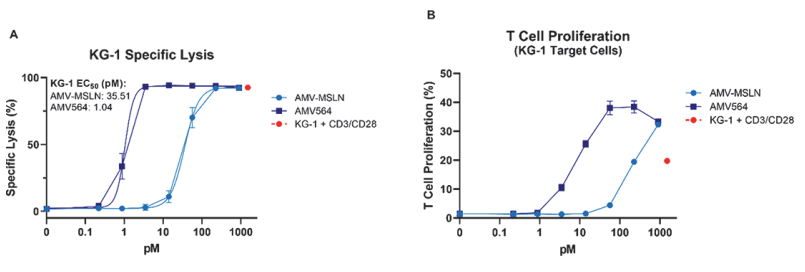

Abstract 849 Figure 2 Cytotoxicity assay using primary T cells demonstrates 'core' potency of platform molecules on the CD33expressing KG-1 cell line. Core potency can be engineered to allow appropriate dosing for TME saturation while maintaining selectivity (2A). T cell proliferation exceeds positive control (CD3/CD28) across expected clinical dose range while maintaining selectivity and T cell viability (2B).

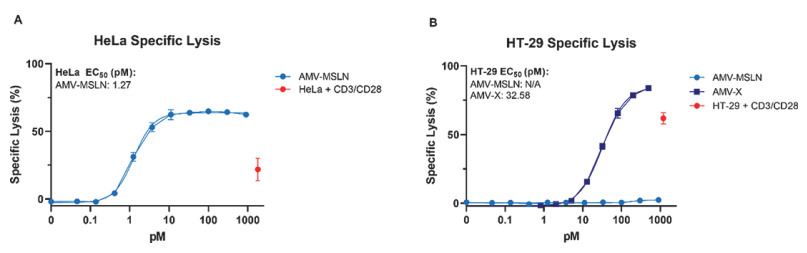

Abstract 849 Figure 3 Bivalent VHH affinity can be engineered to increase or decrease potency depending on the specific target while maintaining selectivity. AMV-MSLN lead demonstrates potent cytotoxic effects on MSLN-high HeLa cells (3A). The same AMV-MSLN molecule does not target MSLN-low HT-29 cells. AMV-X binds a non-MSLN surface antigen on HT-29, demonstrating the potency and specificity of ReSTORE molecules (3B).

Conclusions The clinically validated MDSC-depleting core of the ReSTORE platform molecules allow targeting of specific antigens associated with a variety of solid and hematologic tumor indications. This antigen-specific cytotoxicity of cancer cells occurs in parallel with control of the immunosuppressive MDSC.

\section{REFERENCES}

1. Niharika B Mettu, Alexander Starodub, Sarina Anne Anne Piha-Paul, Raghad Muhsin Abdul-Karim, Gabriel Tinoco, Michael Rahman Shafique, Victoria Smith, Christina Baccei, Patrick Youngwhan Chun. Results of a phase 1 dose-escalation study of AMV564, a novel T-cell engager, alone and in combination with pembrolizumab in patients with relapsed/refractory solid tumors. J Clin Oncol 39,2021 (suppl 15; abstr 2555).

2. Sterling Eckard, Aurelien Sarde, Li Mei, Curtis Ruegg, Patrick Chun, Victoria Smith. Abstract 528: MDSC suppress the T cell repertoire and contribute to a pathologic cytokine milieu in cancer patients. Cancer Res July 12021 (81) (13 Supplement) 528; DOI: 10.1158/1538-7445.AM2021-528

http://dx.doi.org/10.1136/jitc-2021-SITC2021.849 